\title{
Heliospheric modulation of galactic cosmic rays: Effective energy of ground-based detectors
}

\author{
Agnieszka Gil* \\ Institute of Mathematics and Physics, Siedlce University, Stanislawa Konarskiego 2, 08-110 \\ Siedlce, Poland \\ E-mail: gila@uph.edu.pl

\section{Eleanna Asvestari} \\ Department of Physics, University of Helsinki, FIN-00014 Finland and Space Climate Research \\ Unit, University of Oulu, FIN-90014 Finland
}

\section{Gennady A. Kovaltsov}

Ioffe Physical-Technical Institute, Politechnicheskaya st. 26, 194021 St. Petersburg, Russia

\section{Ilya Usoskin}

Space Climate Research Unit and Sodankylä Geophysical Observatory, University of Oulu, FIN-90014 Finland

\begin{abstract}
Variability of Galactic cosmic ray (GCR) is often expressed in terms of the modulation potential, which is typically assessed using energy-integrating ground-based detectors, such as neutron monitors (NMs) for the last decades or cosmogenic isotopes on the time scales of centuries and millennia. In order to estimate the energy dependence of the GCR variability we re-assess here the effective energy $E_{\text {eff }}$ of each type of detector, which is defined so that the variability of the GCR particles at this energy is equal to that of the detector's count rate. We found that $E_{\text {eff }}$ is 11-12 GeV/nuc for the standard polar sea-level neutron monitor, but it is essentially smaller for cosmogenic isotopes, being 6-7 GeV/nuc for ${ }^{14} \mathrm{C}$ and 5.5-6 GeV/nuc for ${ }^{10} \mathrm{Be}$, respectively. It is also discussed that this effective energy is robustly defined and is hardly dependent on the primary assumptions on the local interstellar spectrum (LIS) of GCR.
\end{abstract}

35th International Cosmic Ray Conference - ICRC2017

10-20 July, 2017

Bexco, Busan, Korea

\footnotetext{
* Speaker.
} 


\section{Introduction}

Variability of Galactic cosmic rays (GCR) near Earth is caused by the heliospheric modulation [1] of the local interstellar spectrum (LIS) which can be assumed roughly constant on the time scale shorter than a million of years. Here we use, following [2] the LIS model by Vos and Potgieter [3]. In an ideal case, the spectrum of GCR can be directly measured by balloon- or space-borne instruments, including precise instruments as PAMELA [4] or AMS [5] for the last decade. The GCR variability is monitored by the global network of ground-based neutron monitors since 1951 [6] and by ionization chambers since the mid-1930s; the latter may however contain a long-term drift [7]. Before that, GCR variability can be estimated only by measurements of cosmogenic radioisotopes in natural archives $[8,9]$. The GCR modulation is often described via the force-field model $[10,11]$ which offers a single time-variable parameter, the modulation potential [11].

Here we re-define the method to estimate GCR variability by means of using energy-integration ground-based detectors, including both the NM and the method of cosmogenic isotopes ${ }^{10} \mathrm{Be}$ and ${ }^{14} \mathrm{C}$ stored in natural archives.

\section{Cosmic ray detection}

The Earth's atmosphere is thick, and energetic cosmic ray particles cause a complicated nucleonicmuon-electromagnetic cascade, whose secondaries are registered by ground-based detectors, which are energy-integrating devices.

\subsection{Neutron monitor}

A neutron monitor (NM) is a standard detector to register GCR variability on the time scales from an hour up to a solar cycle. The world-wide network (several tens of the standard-design detectors around the Globe - see http://www.nmdb.eu) exists since 1951. Count rate of a NM is caused by the local flux of secondary nucleons. It can be presented as follows (see, e.g., [6]):

$$
N(t)=\sum_{i=1}^{m} \int_{E_{\mathrm{c}, i}}^{\infty} Y_{i}(E) \cdot J_{i}(E, t), d E
$$

where summation is over $i$-th species of the primary CR (protons, $\alpha$-particles, heavier nuclei), $J(E, t)$ is the energy spectrum of CR outside the Earth's magnetosphere, $Y(E)$ is the yield function of a NM which depends of kinetic energy, $E$, of the primary CR particles, and $E_{\mathrm{c}}$ is the CR particle's kinetic energy corresponding to the geomagnetic cutoff rigidity $P_{\mathrm{c}}$ as

$$
E_{\mathrm{c}, i}=\sqrt{\left(\frac{Z_{i}}{A_{i}}\right)^{2} P_{\mathrm{c}}^{2}+E_{0}^{2}}-E_{0},
$$

where $E_{0}=0.938 \mathrm{GeV} /$ nuc is the rest mass of a proton.

The yield function of a standard NM (a polar sea-level of the type 1-NM64) we use here, has been calculated elsewhere [12]. 


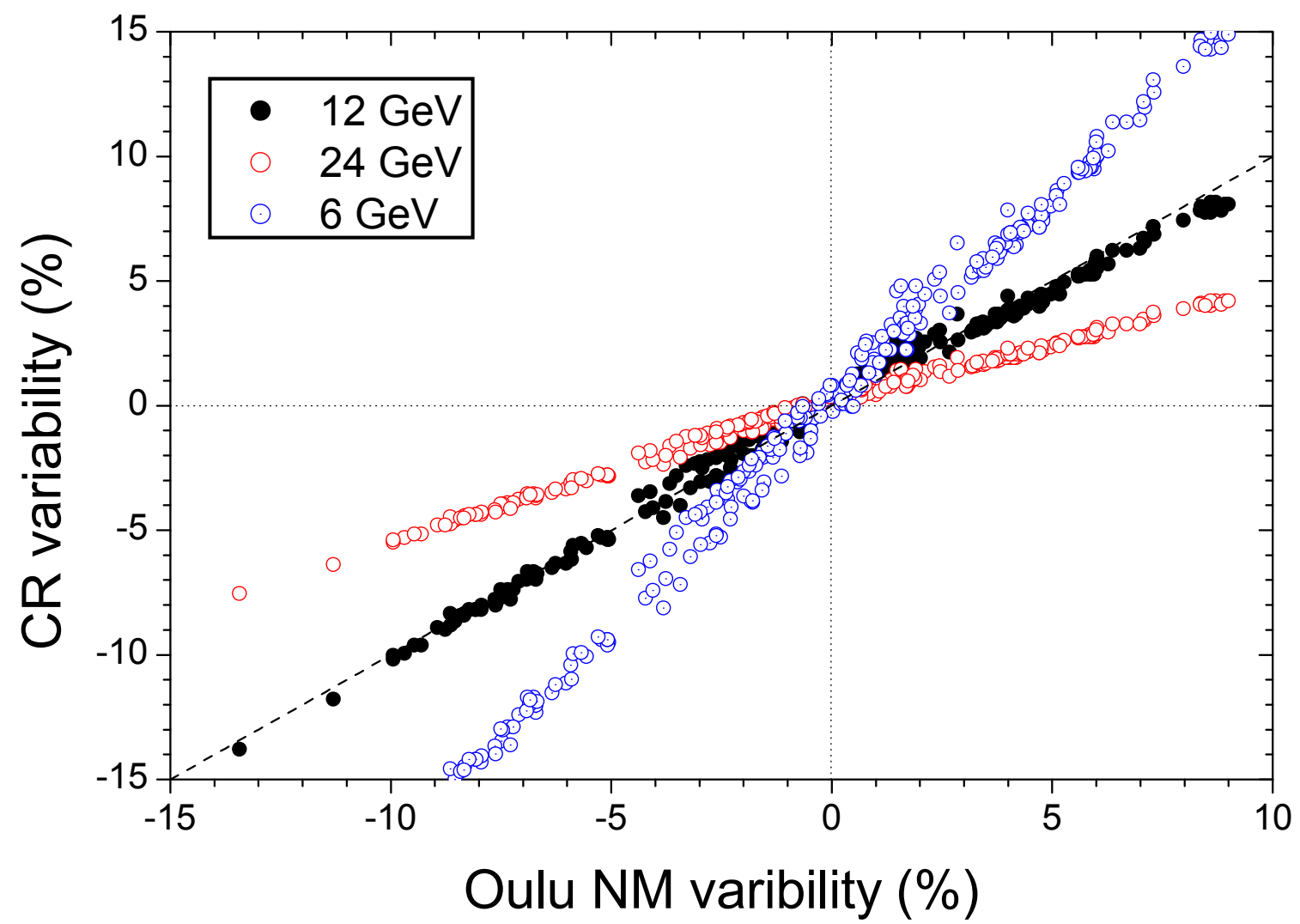

Figure 1: Relative variability (in \% to the mean level) for the period 1993-2016 of the GCR flux at fixed energy (as denoted in the legend) as a function of the the Oulu NM count rate variability (X-axis).

\subsection{Radiocarbon ${ }^{14} \mathrm{C}$}

Radiocarbon ${ }^{14} \mathrm{C}$ is produced in the Earth's atmosphere as a result of the capture of a thermal neutron by atmospheric nitrogen. Atmospheric neutrons are secondary particles of the nucleonic cascade and thus, production of radiocarbon is defined by the GCR flux at any moment. After production, radiocarbon gets oxidized to carbon dioxide and takes part, as gas, in the global carbon cycle. Accordingly, it is globally mixed in the terrestrial system. Production of ${ }^{14} \mathrm{C}$ can be also described by Equation 2.1 (with the corresponding yield function $Y$ ), which should be additionally integrated over the Globe. Here we apply the yield function of ${ }^{14} \mathrm{C}$ production as in $[13,14]$.

\subsection{Cosmogenic ${ }^{10} \mathrm{Be}$}

Cosmogenic isotope ${ }^{10} \mathrm{Be}$ is produced as a result of spallation of nitrogen and oxygen nuclei in the development of a nucleonic cascade in the atmosphere. Then beryllium gets attached to atmospheric aerosols, which leads to relatively fast sedimentation and deposition upon surface without being involved into a global cycle. This process can be reasonably well modelled by atmospheric dynamical models (e.g., [15, 16]). Here we apply the parametrization [15] using transport coefficients of six large atmospheric 'boxes' and the production model of [14]. 


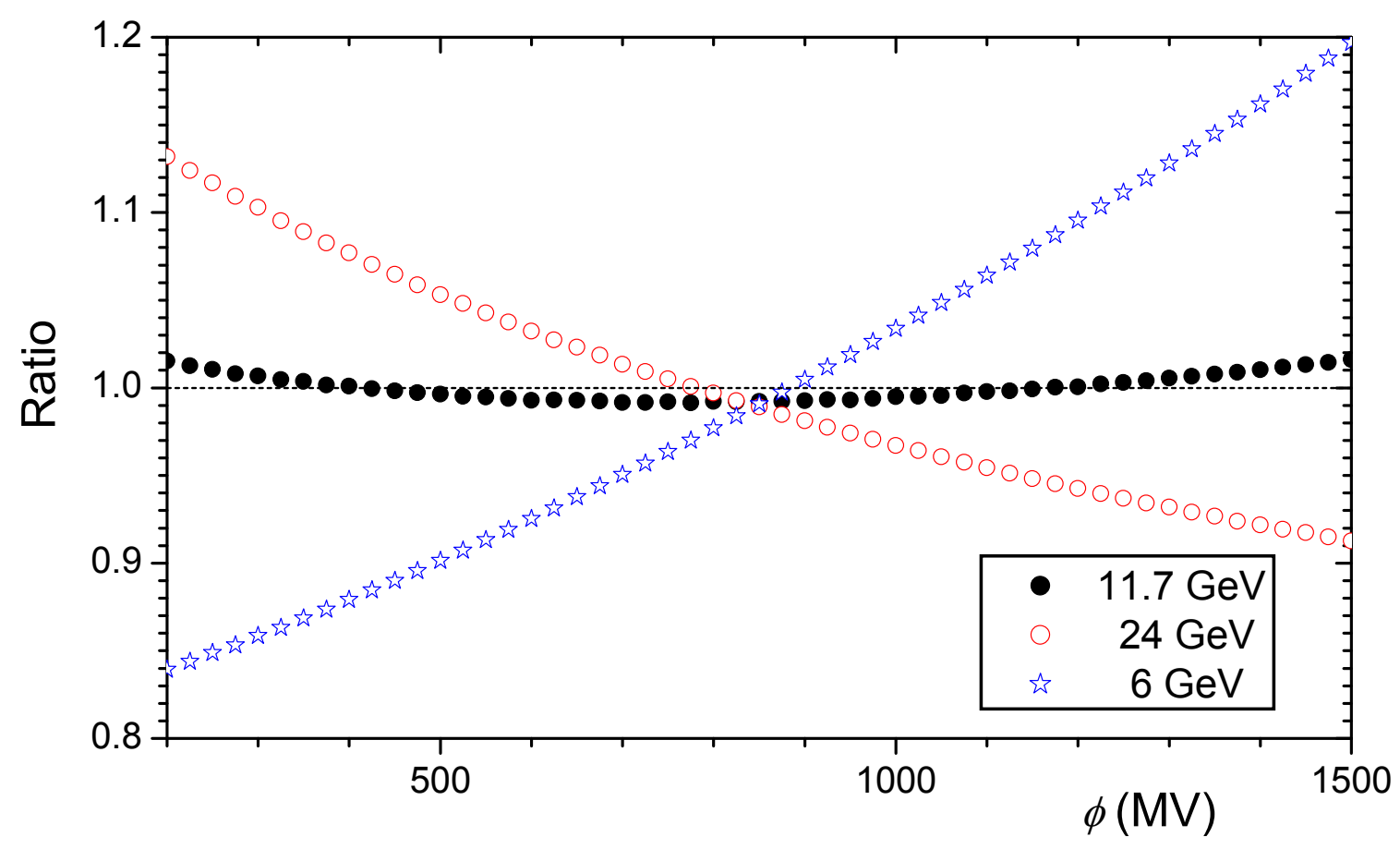

Figure 2: Ratio of the polar NM count rate to the flux of GCR protons with given energy (as denoted in the legend) as function of the modulation potential $\phi$. All curves are normalized to the unity in the range of 200-1500 MV, as indicated by the horizontal dashed line.

\section{Effective energy}

It is typical to describe an energy-integrating CR detector by its 'effective energy', which may have different meanings, such as: fixed energy/rigidity (10 GV for a NM, [17]), median energy $[18,19]$, maximum of the differential response function [20], or the integral effective energy [21]. However, these concepts are not intuitively understandable and may fluctuate with a solar cycle. Here we present a concept of the effective energy $E_{\text {eff }}$ such that the variability of the GCR flux at this energy is directly proportional to that of the detector's count rate.

Figure 1 depicts the temporal profile of the data of Oulu NM since 1993, along with the variability of the flux of GCR protons at fixed energy of 6,12 and $24 \mathrm{GeV}$. One can see that while higher-energy protons $(24 \mathrm{GeV})$ exhibit too low variability of $\pm 4 \%$ and lower-energy protons too high variability $( \pm 15 \%)$, protons with $12 \mathrm{GeV}$ energy almost exactly correspond to the variability of Oulu NM count rate $( \pm 10 \%)$. Thus, the effective energy of Oulu NM is close to $12 \mathrm{GeV} /$ nuc.

We quantify the effective energy $E_{\text {eff }}$ as that which makes the ratio $R(E, \phi)=N(\phi) / J(E, \phi)$ nearly constant (between $\phi 200-1500 \mathrm{MV}$ ), as illustrated in Figure 2. The value $E_{\text {eff }}=11.7$ $\mathrm{GeV} /$ nuc keeps $R$ constant ( $\pm 1 \%$ ), while too high or too low energy leads to an essential trend in the relation. This value is considered as the effective energy for a polar NM. The value of $E_{\text {eff }}$ does not depend, by construction, on the solar cycle, but it changes with the geomagnetic cutoff rigidity, as shown in Figure 3.

Similarly, the effective energy can be defined also for cosmogenic isotopes. It slightly depends on the geomagnetic dipole moment $M$ and LIS models. For the LIS adopted here [3] and the modern 


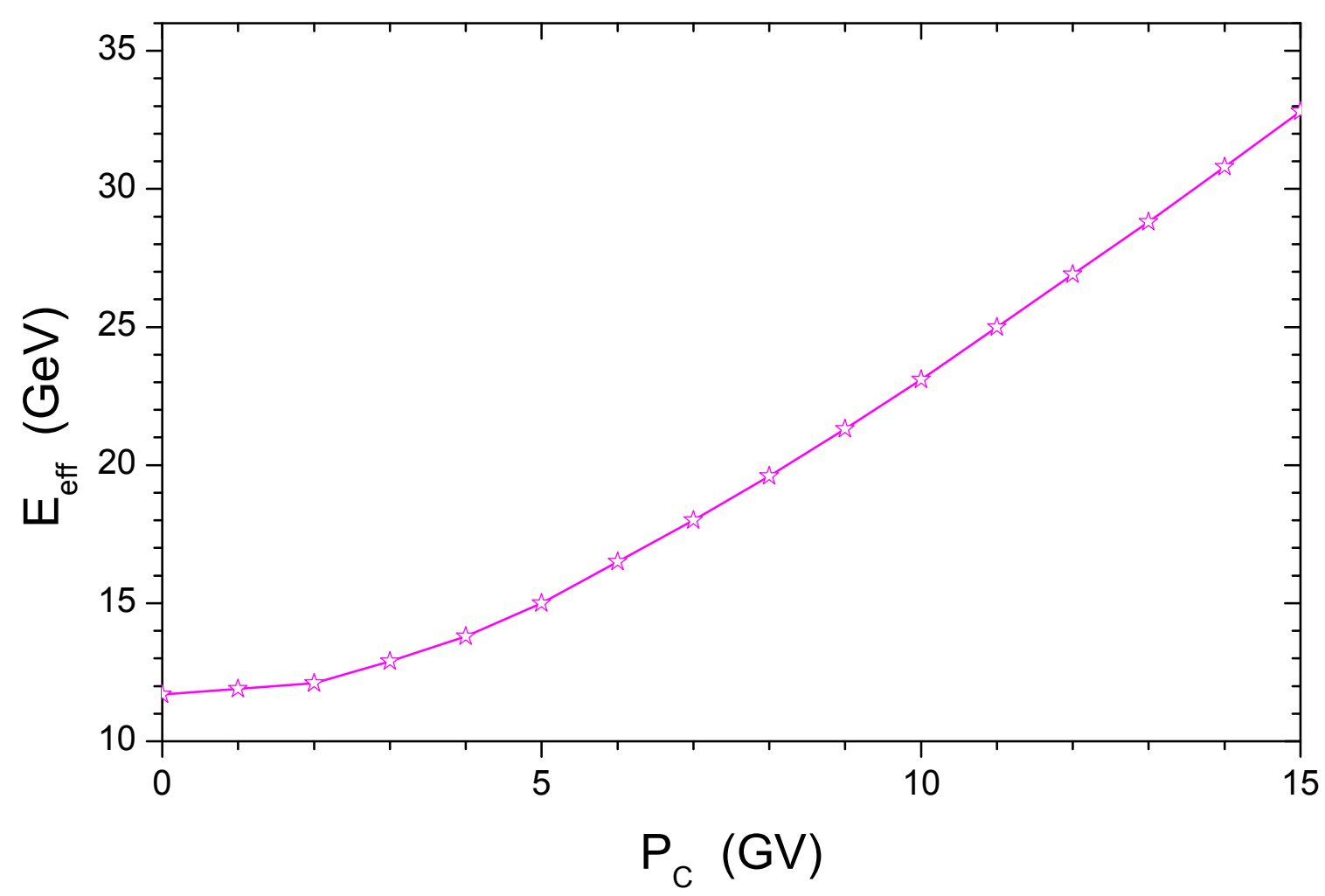

Figure 3: Dependence of the effective energy $E_{\text {eff }}$ of a standard sea-level NM on the geomagnetic rigidity cutoff.

geomagnetic epoch, the effective energy appears to be equal to $6.6 \mathrm{GeV} / \mathrm{nuc}$ (ranging between $6.2-$ $7.5 \mathrm{GeV} /$ nuc depending on the exact LIS and $M$ ) for global ${ }^{14} \mathrm{C}$ and to $5.5 \mathrm{GeV} /$ nuc $(5.2-6.3$ $\mathrm{GeV} / \mathrm{Nuc}$ ) for polar ${ }^{10} \mathrm{Be}$. The effective energy for cosmogenic isotopes is nearly half of that for the NM implying that they are sensitive to less energetic cosmic rays than even a polar NM. It should be noted that by definition this effective energy only relates to the GCR variability. The effective energy for SEPs is significantly lower, of the order of $200 \mathrm{MeV}$ for ${ }^{14} \mathrm{C}$ and ${ }^{10} \mathrm{Be}[22]$, and $800 \mathrm{MeV}$ for NM [23], because of much softer energy spectrum of SEP.

\section{Conclusions}

We have offered a new definition of the effective energy of GCR for ground-based measurements. It is defined as the energy, at which GCR protons have the same relative variability as the count rate of the detector. This effective energy appears to vary for NMs from 11-12 GeV/nuc for polar up to $33 \mathrm{GeV} / \mathrm{nuc}$ for the equatorial location. On the other hand, cosmogenic isotopes are responsive to cosmic rays of lower energy, with the effective energy being $5-6 \mathrm{GeV} /$ nuc for ${ }^{10} \mathrm{Be}$ and 6-7 GeV/nuc for ${ }^{14} \mathrm{C}$. This result offers a simple way to interpret the data of GCR variability obtained by different methods. 


\section{Acknowledgements}

This work was supported by the Center of Excellence ReSoLVE (project No. 272157). A.G. acknowledges The Polish National Science Centre, decision number DEC-2016/22/E/HS5/00406.

\section{References}

[1] M. Potgieter, Solar Modulation of Cosmic Rays, Living Rev. Solar Phys. 10 (2013) 3, [arXiv:1306.4421].

[2] I. G. Usoskin, A. Gil, G. Kovaltsov, A. Mishev, and V. Mikhailov, Heliospheric modulation of cosmic rays during the neutron monitor era: Calibration using pamela data for 2006-2010, J. Geophys. Res. Space Phys. 122 (2017) 3875-3887. 2016JA023819.

[3] E. E. Vos and M. S. Potgieter, New Modeling of Galactic Proton Modulation during the Minimum of Solar Cycle 23/24, Astrophys. J. 815 (2015) 119.

[4] O. Adriani, G. C. Barbarino, G. A. Bazilevskaya, et al., Time Dependence of the Proton Flux Measured by PAMELA during the 2006 July-2009 December Solar Minimum, Astrophys. J. 765 (2013) 91.

[5] M. Aguilar, G. Alberti, B. Alpat, A. Alvino, G. Ambrosi, K. Andeen, H. Anderhub, L. Arruda, P. Azzarello, A. Bachlechner, and et al., First Result from the Alpha Magnetic Spectrometer on the International Space Station: Precision Measurement of the Positron Fraction in Primary Cosmic Rays of 0.5-350 GeV, Phys. Rev. Lett. 110 (2013), no. 14141102.

[6] I. G. Usoskin, K. Alanko-Huotari, G. A. Kovaltsov, and K. Mursula, Heliospheric modulation of cosmic rays: Monthly reconstruction for 1951-2004, J. Geophys. Res. 110 (2005) A12108.

[7] K. McCracken and J. Beer, Long-term changes in the cosmic ray intensity at Earth, 1428-2005, J. Geophys. Res. 112 (2007), no. A11 A10101.

[8] J. Beer, K. McCracken, and R. von Steiger, Cosmogenic Radionuclides: Theory and Applications in the Terrestrial and Space Environments. Springer, Berlin, 2012.

[9] I. G. Usoskin, A History of Solar Activity over Millennia, Living Rev. Solar Phys. 14 (2017) 3.

[10] L. Gleeson and W. Axford, Solar modulation of galactic cosmic rays, Astrophys. J. 154 (1968) 1011-1026.

[11] R. Caballero-Lopez and H. Moraal, Limitations of the force field equation to describe cosmic ray modulation, J. Geophys. Res. 109 (2004) A01101.

[12] A. L. Mishev, I. G. Usoskin, and G. A. Kovaltsov, Neutron monitor yield function: New improved computations, J. Geophys. Res. 118 (2013) 2783-2788.

[13] G. Kovaltsov, A. Mishev, and I. Usoskin, A new model of cosmogenic production of radiocarbon ${ }^{14} C$ in the atmosphere, Earth Planet. Sci. Lett. 337 (2012) 114-120.

[14] S. V. Poluianov, G. A. Kovaltsov, A. L. Mishev, and I. G. Usoskin, Production of cosmogenic isotopes ${ }^{7} \mathrm{Be},{ }^{10} \mathrm{Be},{ }^{14} \mathrm{C},{ }^{22} \mathrm{Na}$, and ${ }^{36} \mathrm{Cl}$ in the atmosphere: Altitudinal profiles of yield functions, J. Geophys. Res. (Atm.) 121 (2016) 8125-8136, [arXiv: 1606.0589 ].

[15] U. Heikkilä, J. Beer, and J. Feichter, Meridional transport and deposition of atmospheric ${ }^{10}$ Be, Atmos. Chem. Phys. 9 (2009) 515-527. 
[16] T. Sukhodolov, I. Usoskin, E. Rozanov, E. Asvestari, W. Ball, M. Curran, H. Fischer, G. Kovaltsov, F. Miyake, T. Peter, C. Plummer, W. Schmutz, M. Severi, and R. Traversi, Atmospheric impacts of the strongest known solar particle storm of 775 AD, Sci. Rep. 7 (2017) 45257.

[17] A. Belov, Large Scale Modulation: View From the Earth, Space Sci. Rev. 93 (2000) 79-105.

[18] H. S. Ahluwalia and L. I. Dorman, Transverse cosmic ray gradients in the heliosphere and the solar diurnal anisotropy, J. Geophys. Res. 102 (1997) 17433-17444.

[19] T. Jämsén, I. G. Usoskin, T. Räihä, J. Sarkamo, and G. A. Kovaltsov, Case study of Forbush decreases: Energy dependence of the recovery, Adv. Space Res. 40 (2007) 342-347.

[20] K. McCracken, F. McDonald, J. Beer, G. Raisbeck, and F. Yiou, A phenomenological study of the long-term cosmic ray modulation, 850-1958 ad, J. Geophys. Res. 109 (2004), no. A18 12103.

[21] K. Alanko, I. G. Usoskin, K. Mursula, and G. A. Kovaltsov, Heliospheric modulation strength: Effective neutron monitor energy, Adv. Space Res. 32 (2003) 615-620.

[22] G. A. Kovaltsov, I. G. Usoskin, E. W. Cliver, W. F. Dietrich, and A. J. Tylka, Fluence Ordering of Solar Energetic Proton Events Using Cosmogenic Radionuclide Data, Solar Phys. 289 (2014) 4691-4700.

[23] A. Asvestari, W. T., A. Gil, I. Usoskin, G. Kovaltsov, V. Mikhailov, and A. Mayorov, Analysis of ground level enhancements (gle): Extreme solar energetic particle events have hard spectra, Adv. Space Res. (2017) (in press). 